\title{
Use of Biomarkers and Multigene Assays in Breast Cancer
}

\author{
Presented by Lee S. Schwartzberg, MD
}

\begin{abstract}
All patients with early breast cancer should be classified by subgroup through testing estrogen receptor, progesterone receptor, and HER2 status, with or without Ki-67 proliferation percentage. Genomic expression profiling aids clinical decision-making in most patients, because most are estrogen receptor-positive. The commercially available classifiers are prognostic for distant recurrence in node-negative and also node-positive patients. Patients at genomically low risk have excellent 5-year outcomes with endocrine therapy alone. Some assays also predict for benefit from adjuvant endocrine therapy and chemotherapy in node-negative and node-positive patients, but with varying levels of evidence. Genomic classifiers are also prognostic of late relapse and may predict benefit from extended adjuvant endocrine therapy. J Natl Compr Canc Netw 2017;15(5.5):676-678
\end{abstract}

\section{Use of Biomarkers and Multigene Assays in Breast Cancer}

Genomic expression profiling (GEP) has come of age as a means of providing prognostic and predictive information for patients with estrogen receptor (ER)positive early-stage breast cancer, and can greatly aid in treatment decision-making, according to Lee S. Schwartzberg, MD, Executive Director, West Cancer Center, which is affiliated with the University of Tennessee. Dr. Schwartzberg described the evidence supporting the use of commercially available assays at the NCCN 22nd Annual Conference.

\section{Using GEP to Tailor Treatment}

For patients with hormone receptor-positive, HER2negative disease-some $70 \%$ of patients with early-stage breast cancer-multigene assays can aid in decisionmaking. "This is where we can actually cure women with occult micrometastatic disease," Dr. Schwartzberg said.

Presented by Lee S. Schwartzberg, MD, West Cancer Center, and Division of Hematology/Oncology, University of Tennessee Health Science Center, Memphis, Tennessee.

Dr. Schwartzberg has disclosed that he has received consulting fees and honoraria from Amgen Inc., Genentech, Inc., Genomic Health, Inc.

Pfizer Inc., Tesaro, Inc., and has served as a scientific advisor for Bristol-

Myers Squibb Company, Caris Life Sciences, Helsinn Group, and Merck

\& Co., Inc.

Correspondence: Lee S. Schwartzberg, MD, West Cancer Center

7945 Wolf River Boulevard, Memphis, TN 38138.

E-mail: Ischwartzberg@westclinic.com
At least 6 genomic classifiers are now commercially available: the 21-gene recurrence score (RS) assay, 70 gene assay, the Breast Cancer Index, 5-gene immunohistochemistry assay, 8-gene assay, and 50-gene assay.

\section{It Started With the 21-Gene RS}

"The assays are all prognostic, and in general they are also all predictive of endocrine therapy benefit," Dr. Schwartzberg indicated.

The most widely used assay is the RS, which incorporates 16 informative cancer genes and 5 reference genes and is highly prognostic for outcomes. The NSABP-14 trial validated its usefulness by showing that 10 -year recurrence rates corresponded significantly with risk score $(P<.001) .{ }^{1}$ Recurrence rates were $6.8 \%$ for patients with a low RS $(<11), 14.3 \%$ for intermediate RS (18-30), and 30.6\% for high RS ( $\geq 31){ }^{1}$

Similar patterns were shown for the other GEP tests. For example, the 70-gene assay classified patients into "good" and "poor" gene signature groups, whose respective metastases-free survival rates at 10 years were $87 \%$ and $44 \%$, respectively, and overall survival rates were $97 \%$ and $50 \%$, respectively. ${ }^{2}$

The TAILORx trial provided a compelling prospective analysis. ${ }^{3}$ In this study, $>10,000$ patients were enrolled, of whom $16 \%$ had a RS of 0 to 10 and received endocrine therapy alone. ${ }^{3}$ This low-risk group had a $<1 \%$ distant recurrence rate at 5 years. Other end points for 
this group of patients were equally good, including freedom from distant recurrence at 5 years $(99.3 \%)$, freedom from recurrence $(98.7 \%)$, invasive diseasefree survival (93.8\%), and overall survival (98.0\%).

"This is still early follow-up, because the natural history of ER-positive breast cancer is long, but these are obviously outstanding results that give us comfort," Dr. Schwartzberg commented.

\section{GEP for Predicting Treatment Benefit}

"Many clinicians feel that GEP for the prediction of chemotherapy benefit may be the most impactful way we can use these tests," Dr. Schwartzberg remarked.

In NSABP B-14, the benefit of tamoxifen (vs placebo) in patients with ER-positive cancer was seen in the low and intermediate RS groups, but not the high RS group. Discrimination by RS groups was extended to the addition of adjuvant chemotherapy to tamoxifen in the follow-up B-20 trial. ${ }^{4}$ Conversely to the effect of endocrine therapy, chemotherapy clearly reduced 10-year distant disease-free recurrence rates in the high RS group, with no clear benefit in the low or intermediate RS groups. Results from the prospective randomized portion of the TAILORx trial, comparing endocrine therapy alone to endocrine and chemotherapy in an intermediate RS group, are eagerly awaited.

Most recently, results were published for the prospective MINDACT trial, which also tested the benefit of chemotherapy by GEP prospectively. ${ }^{5}$ Among 6,693 patients with early-stage breast cancer, the researchers compared the ability of the 70-gene signature versus traditional clinical and biological criteria (using Adjuvant! Online) to identify patients who can safely forego chemotherapy without compromising distant metastasis-free survival (DMFS).

The population was subdivided based on concordance or discordance between the test results: low risk by both risk assessments, high risk by both, or discordant. Adjuvant chemotherapy was prescribed to patients who were high risk by both tests and withheld for those considered low risk by both. Discordant patients were randomly assigned to chemotherapy or not (but could receive endocrine therapy). DMFS was $98 \%$ among patients who were low risk by both assessments; $90.6 \%$ for patients at high risk by both and who received chemotherapy; and approximately $95 \%$ for both the discordant groups.
In the randomized cohort, the 5-year DMFS was $94.7 \%$ for patients at high risk clinically but low risk by the 70 -gene signature who did not receive chemotherapy, meeting the study's end point. MINDACT was not powered to determine whether chemotherapy benefited the discordant groups, but there was a nonsignificant trend for survival without distant metastases in patients with high clinical but low genomic risk receiving chemotherapy (hazard ratio [HR], $0.65 ; P=.11)$. In contrast, patients with low clinical but high genomic risk had DMFS curves that overlapped with or without chemotherapy.

"Patients with low clinical and low genomic risk using the 70-gene assay appeared to be comparable to patients with 21 -gene $\mathrm{RS}<11$ in TAILORx. They had a $98 \%$ to $99 \%$ 5-year rate of no distant metastases, so that's a very good prognosis group," Dr. Schwartzberg commented.

Results for the TAILORx intermediate group randomized to chemotherapy or no chemotherapy will help determine the extent of chemotherapy benefit. Until these results become known, a population-based analysis from the SEER database of 40,000 node-negative patients assayed with the 21-gene RS provides informative information. At 5 years, breast cancer-specific mortality was $0.4 \%$ in the low RS group, $1.4 \%$ for the intermediate RS group, and $4.4 \%$ in the high RS group. " "In the SEER database, the vast majority of low RS patients received endocrine therapy alone, some in the intermediate group received chemotherapy, and the large majority of high RS patients received both endocrine therapy and chemotherapy," he said.

\section{RS in Node-Positive Patients}

Lymph node positivity is now recognized to reflect "a continuous biology of breast cancer," with greater involvement proportionally associated with worse outcomes. The 21-gene RS also addresses this issue and has been found to be prognostic in node-positive and node-negative cancer, based on studies showing that patients with node-positive cancer with high RS had worse outcomes with endocrine therapy alone.

Whether RS is also predictive in patients with node-positive disease is not yet fully established, although results from SWOG-8814 suggest so. ${ }^{7}$ Chemotherapy was associated with significantly improved breast cancer-specific survival at 10 years 
(73\%) versus tamoxifen alone $(54 \%)$ in patients with high RS $(P<.033)$, but not in those with low or intermediate RS disease. Answers may come from the RxPONDER trial in 8,800 women with ER-positive breast cancer, 1 to 3 positive nodes, and RS $<25$ who are being randomized to endocrine therapy versus endocrine therapy and chemotherapy.

\section{Late Recurrence, New AJCC Staging}

GEP can also be informative about the risk of late recurrences, common in ER-positive disease. An analysis by the Early Breast Cancer Trialists' Collaborative Group confirmed that, to 20 years, recurrence rates after tamoxifen exposure have not plateaued. ${ }^{8}$ Analysis of each of the genomic classifiers shows, coupled with clinical factors, they can stratify patients into lower and higher risk of later relapse between years 5 through 10 after diagnosis. The Breast Cancer Index also appears to have some predictive value for prolonged endocrine therapy.

As a fundamental change, the latest cancer staging manual from AJCC adds the 21-gene RS to conventional factors included in determining disease stage. In particular, $\mathrm{RS}<11$, even in a patient with T2N0 grade 3 tumor, signifies stage IA because of the excellent prognosis in this subset.

\section{How the NCCN Guidelines Incorporate RS}

The NCCN Clinical Practice Guidelines in Oncology (NCCN Guidelines) for Breast Cancer ${ }^{9}$ recommend that for patients with hormone receptor-positive, HER2-negative, node-negative disease with tumors $>0.5 \mathrm{~cm}$, the 21 -gene RS assay should be considered. For RS $<18$, endocrine therapy alone is recommended. For intermediate RS (18-30), endocrine therapy alone or with chemotherapy is acceptable. High RS ( $\geq 31)$ warrants both approaches.

Footnotes to these recommendations indicate that the assay can be considered for patients with 1 to 3 positive nodes and that other GEP assays "are also prognostic," he said, "but are backed by less evidence for prediction of a chemotherapy benefit."

\section{References}

1. Paik $S$, Shak $S$, Tang $G$, et al. A multigene assay to predict recurrence of tamoxifen-treated, node-negative breast cancer. N Engl J Med 2004:351:2817-2826.

2. Van't Veer LJ, Dai H, van de Vijver MJ, et al. Gene expression profiling predicts clinical outcome of breast cancer. Nature 2002;415:530-536.

3. Sparano JA, Gray RJ, Makower DF, et al. Prospective validation of a 21. gene expression assay in breast cancer. N Engl J Med 2015;373:2005-2014.

4. Paik S, Tang G, Shak S, et al. Gene expression and benefit of chemotherapy in women with node-negative, estrogen receptor-positive breast cancer. J Clin Oncol 2006;24:3726-3734.

5. Cardoso F, van't Veer LJ, Bogaerts J, et al. 70-gene signature as an aid to treatment decisions in early-stage breast cancer. N Engl J Med 2016;375:717-729.

6. Petkov VI, Miller DP, Howlader N, et al. Breast-cancer-specific mortality in patients treated based on the 21-gene assay: a SEER population-based study. Available at: https://www.nature.com/articles/npjbcancer201617. Accessed April 11, 2017.

7. Albain KS, Barlow WE, Shak S, et al. Prognostic and predictive value of the 21-gene recurrence score assay in postmenopausal women with nodepositive, oestrogen-receptor-positive breast cancer on chemotherapy: a retrospective analysis of a randomised trial. Lancet Oncol 2010;11:55-65.

8. Pan H, Gray RG, Davies C, et al. Predictors of recurrence during years 5-14 in 46,138 women with ER+ breast cancer allocated 5 years only of endocrine therapy (ET) [abstract]. J Clin Oncol 2016;34(Suppl):Abstract 505.

9. Gradishar WJ, Anderson BO, Balassanian R, et al. NCCN Clinical Practice Guidelines in Oncology for Breast Cancer, Version 1.2017. Accessed April 5, 2017. To view the most recent version of these guidelines, visit NCCN org 